\title{
Nonlinearities of an Aircraft Piccolo Tube: Identification and Modeling
}

\author{
T. Dossogne ${ }^{1}$, P. Trillet ${ }^{1}$, M. Schoukens ${ }^{2}$, B. Bernay ${ }^{3}$, J.P. Noël ${ }^{1}$ and G. Kerschen ${ }^{1}$ \\ ${ }^{1}$ Space Structures and Systems Lab, Aerospace and Mechanical \\ Engineering Department, University of Liège, Belgium \\ ${ }^{2}$ Control Systems Group, Department of Electrical Engineering, \\ Eindhoven University of Technology, The Netherlands \\ ${ }^{3}$ SONACA SA, Gosselies, Belgium \\ Email: tdossogne@uliege.be, pierre.trillet@gmail.com,m.schoukens@tue.nl,jp.noel@uliege.be \\ bruno.bernay@sonaca.com, g.kerschen@uliege.be
}

\begin{abstract}
Piccolo tubes are parts of aircraft wings anti-icing system and consist of titanium pipes inserted into the internal structure of the slat. Due to differential thermal expansion, clearances between the tube and its support are unavoidable and cause the overall system to exhibit highly nonlinear behavior, resulting from impacts and friction. This paper addresses the identification and modeling of the nonlinearities present in the slat-Piccolo tube connection. The complete identification procedure, from nonlinearity detection and characterization to parameter estimation, is carried out based upon sine-sweep measurements. The use of several techniques, such as the acceleration surface method, enables to understand the complex dynamics of the Piccolo tube and build a reliable model of its nonlinearities. In particular, the parameters of nonsmooth nonlinear stiffness and damping mechanisms are estimated. The nonlinear model is finally validated on standard qualification tests for airborne equipments.
\end{abstract}

Keywords: Nonlinear system identification, Finite element model updating, Finite element model upgrading, Aircraft anti-icing system, Piccolo tube, Acceleration Surface Method (ASM), Vibroimpact, Contact force model.

\section{Introduction}

Subsystems of commercial aircraft are submitted to rigorous qualification processes imposed by aeronautical norms. In this context, the need for trustworthy finite element (FE) models is crucial. However, the growing occurrence of nonlinear structural mechanisms makes the validation of numerical models more arduous, if not impossible, by using only linear analysis. Upgrading the model with nonlinear elements and performing a complete identification of their parameters is therefore required.

A typical case of such a subsystem is the Piccolo tube, which is part of the hot-air, anti-icing system. They are widely used in commercial aircraft as they represent a simple and efficient solution to address the accumulation of ice on the wings, which remains a critical issue for the aircraft safety and performance. They consist of titanium pipes inserted in the internal structure of the slat, that distribute some high-temperature air extracted from the engine compressor into the wing leading edge. During the flight, they are hence exposed to a wide range of temperatures, resulting in substantial changes in their dimensions due to thermal expansion. These variations create clearances between the tube and its support and cause the overall system to exhibit nonlinear behavior due to impacts and friction [1]. Linear FE models have proved to be insufficient to reproduce the experimentally observed dynamics of the tube.

The present paper addresses the identification and the validation of a Piccolo tube nonlinear model. Starting from an updated linear FE model, a complete identification methodology is achieved to characterize and estimate nonlinearities. They are modeled as nonlinear elements that reproduce contact forces including dissipation, at the impact location. Measurements from qualification tests are eventually used to validate the proposed nonlinear model. 


\section{$1 \quad$ Linear Finite Element Modeling and Updating}

A finite element model made of linear shell elements is first built using the measured geometry and masses. It is then updated, based on linear modal parameters estimated from data collected during low-level sine-sweep tests. Such tests are assumed to take place in the linear regime as the amount of energy injected in the system is not sufficient to trigger the contact between the different components. Pictures of the experimental setup in clamped configuration (left) as well as a close-up on the nonlinear connection (right) are shown in Figure 1 (a).

\section{Nonlinearity Characterization using the Acceleration Surface Method}

The Acceleration Surface Method (ASM) is a qualitative variant of the well-known Restoring Force Surface method [2]. The ASM offers a fast visualization tool of nonlinear mechanisms. It relies on an approximation of the nonlinear restoring forces by the acceleration signal plotted with a minus sign over the phase plane [3]. The application of the ASM to high-level sine-sweep data enables unveiling the nonsmooth dynamics occurring at the connection between the tube and its support, as illustrated in Figure 1 (b). A piecewise-linear stiffness is observed and an estimation of the physical clearances can be obtained through this plot. Additional nonlinear identification methods, such as the Best Linear Approximation [4], the wavelet transform analysis [5] or the homogeneity test, were successfully applied to quantify the amount of nonlinearity in the system and highlight the presence of dissipation during the impacts.

\section{Estimation of the Nonlinear Contact Forces using the eXtended Acceleration Surface Method}

By means of Craig-Bampton reduction method, a superelement of the linear FE model is created, only retaining the excitation point and the nodes where nonlinear behavior was previously located. Nonlinear elements with functional forms determined from the charaterization step are added between these degrees of freedom, namely a nonlinear spring with a piecewise-linear characteristic to reproduce contact, and a nonlinear damper to model dissipation [6] during the impacts.

An eXtended version of the Acceleration Surface Method (x-ASM) is used to estimate the parameters of the nonlinear contact force model. The x-ASM makes use of the FE model upgraded with nonlinear elements. Starting with initial guesses of the nonlinear parameters, it carries out numerical simulations using a Newmark scheme to reproduce the experimental tests. Similarly to the characterization step, the classical ASM is applied to the simulated data. The values of the nonlinear parameters are then updated, following an optimization algorithm that uses the difference between the numerical and the experimental ASM results as an objective function. Once the $\mathrm{x}$-ASM is achieved, the contact stiffness parameter of the nonlinear spring and the coefficient of restitution of the nonlinear damper in the Piccolo tube are identified.

\section{Validation of the Nonlinear Model}

To validate the updated nonlinear FE model, standard qualification tests imposed by the aeronautical norm RTCA DO160 [7] are performed both on the numerical model and on the experimental setup. The case of fan blade-off, shown in Figure 2, and of windmilling were successfully passed.

\section{Acknowledgements}

The authors T. Dossogne and J.P. Noël are Research Fellow (FRIA fellowship) and Postdoctoral Researcher, respectively, of the Fonds de la Recherche Scientifique - FNRS which is gratefully acknowledged. The author M. Schoukens would also like to acknowledge the financial support of the European Union (ERC starting grant APROCS). 


\section{Bibliography}

[1] Van Der Vorst, R., Magerman, J., Bernay, B., Vandenberk, S. et al. (2013) Vibration Qualification Test of an Aircraft Piccolo Tube Using Multiple-Input-Multiple-Output Control Technology. SAE Technical Paper, 2013-01-2315

[2] Masri S.F. and Caughey T.K. (1979) A nonparametric identification technique for nonlinear dynamic problems. Journal of Applied Mechanics, 46:433447

[3] Dossogne, T. et al. (2015) Nonlinear Ground Vibration Identification of an F-16 Aircraft - Part II Understanding Nonlinear Behaviour in Aerospace Structures Using Sine-sweep Testing. in Proceedings of the International Forum on Aeroelasticity and Structural Dynamics, St-Petersburg, Russia

[4] Pintelon, R. and Schoukens, J. (2001) System Identification: A Frequency Domain Approach IEEE Press, Piscataway, NJ, USA

[5] Noël, J.-P., Renson, L. and Kerschen, G. (2014) Complex Dynamics of a Nonlinear Aerospace Structure: Experimental Identification and Modal Interactions. Journal of Sound and Vibration, 33:2588-2607

[6] Lankarani H.M. and Nikravesh P.E. (1990) A Contact Force Model with Hysteresis Damping for Impact Analysis of Multibody Systems Journal of Mecahnical Design, 112, 369-376

[7] RTCA SC-135 (2010) Environmental Conditions and Test Procedures for Airborn Equipment, RTCA, Washington, USA

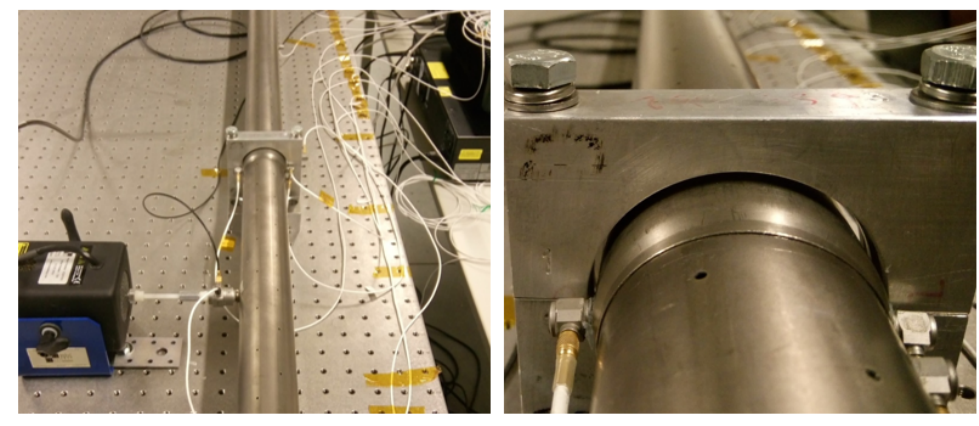

(a)

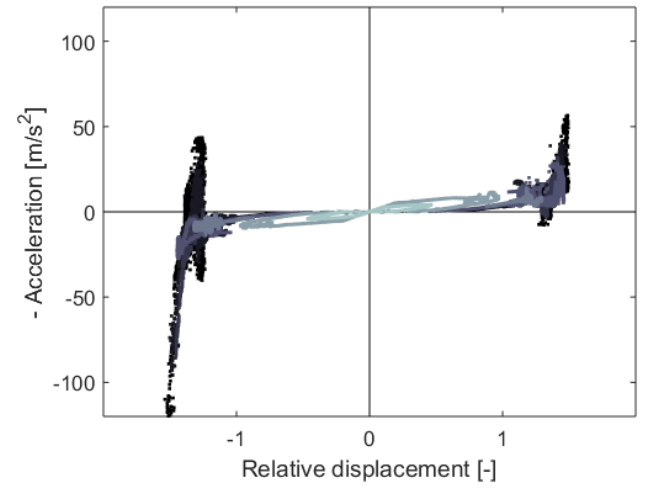

(b)

Figure 1: (a) Experimental setup of the Piccolo tube, SONACA property. Left: setup in clamped configuration - Right: zoom on the nonlinear connection. (b) Qualitative nonlinear stiffness curve of the connection between the Piccolo tube and its support using ASM.
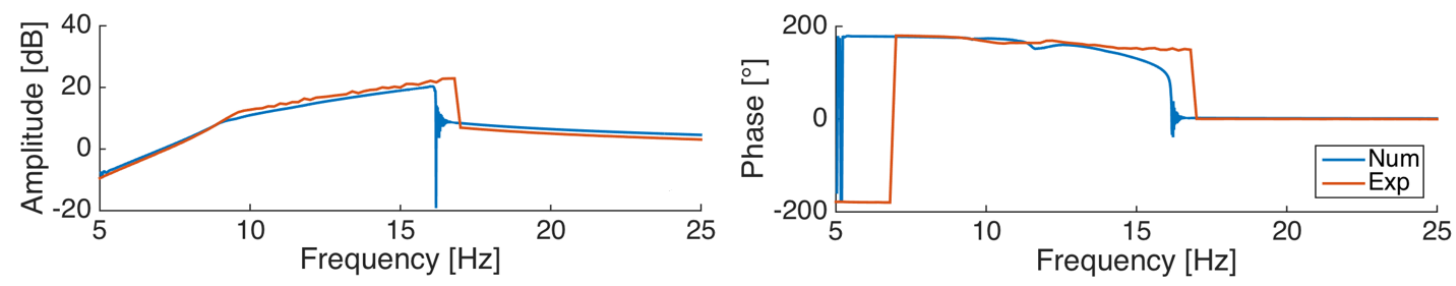

Figure 2: Comparison of the frequency responses in both amplitude and phase between the numerical model (in blue) and the measurement (in orange) during Fan Blade-off tests, focusing on the first vibration mode. 